\title{
Comparison of systems for assuring the eating quality of beef
}

L.J. Farmer, D.J. Devlin, N.F.S. Gault, A.W. Gordon, B.W. Moss, E.L.C. Tolland, I.J. Tollerton

Agri-Food and Biosciences Institute, Belfast, United Kingdom

Email: linda.farmer@afbini.gov.uk

Introduction A number of quality assurance schemes have been developed to grade the eating quality of beef. The UK MLC Blueprint (UK BP) and New Zealand QMark (NZ QM) systems aim to improve eating quality through process control of factors such as carcase suspension, electrical stimulation and ageing. These systems select those carcasses expected to provide consumers with good eating quality. The USDA system classifies beef carcases into quality grades based on the degree of maturity and intramuscular marbling. The Australian MSA system uses process control and carcass characteristics but also classifies individual beef muscles into eating quality grades derived from consumer perceptions, depending on cooking method.

Materials and methods A series of experiments were conducted to assess the role of factors including gender, breed, hanging method, ageing, electrical stimulation/chilling, muscle and position within muscle on eating quality. In total, 192 animals were used, giving 36000 beef samples which were assessed by 6000 consumers. Breed, sex, hot standard carcase weight and EUROP grade were recorded as were hump height, ossification, rib fat, marbling, meat colour, fat colour, ultimate $\mathrm{pH}$ and temperature. Grilled and roasted beef muscles were assessed by consumers who gave it a quality rating: 'unsatisfactory', 'satisfactory everyday quality', 'better than everyday quality' and 'premium' (Farmer et al., 2009). The information recorded enabled the meat to be accorded a classification under several trial quality systems (Table 1). These were very similar to or were modifications of those available internationally. The distribution of the consumer scores for each muscle for each grade was evaluated statistically using a combination of Wald analyses (Genstat) with visual inspection of the distributions to establish the direction of any differences.

Table 1 Grading systems for delivery of good eating quality for NI beef

\begin{tabular}{|c|c|c|c|c|c|}
\hline & MSA-B & USDA & UK BP & UK BP-C & NZ QM \\
\hline \multirow{3}{*}{$\begin{array}{l}\text { Classifications } \\
\text { statistical } \\
\text { evaluation. } \\
\text { Basis } \\
\text { classification } \\
\text { Amendments }\end{array}$} & 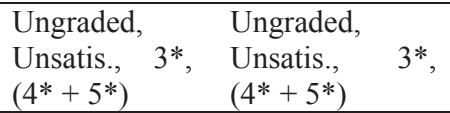 & $\begin{array}{l}\text { Ungraded, Utility, } \\
\text { Standard, Select, } \\
\text { (Choice + Prime) }\end{array}$ & $\begin{array}{l}\text { Ungraded, } \\
\mathrm{BP}, \mathrm{BP}+21 \mathrm{~d}\end{array}$ & $\begin{array}{l}\text { Ungraded, } \\
\mathrm{BP}, \mathrm{BP}+21 \mathrm{~d}\end{array}$ & $\begin{array}{l}\text { Ungraded, } \\
\text { QMark }\end{array}$ \\
\hline & $\begin{array}{l}\text { Processing \& animal data, } \\
\text { muscle \& cooking method }\end{array}$ & $\begin{array}{l}\text { Maturity and } \\
\text { marbling }\end{array}$ & $\begin{array}{l}\text { Processing \& } \\
\text { EUROP grade }\end{array}$ & animal data & $\begin{array}{l}\text { Processing } \\
\text { data }\end{array}$ \\
\hline & Bulls included & - & - & 'O' included & - \\
\hline
\end{tabular}

Results and Discussion Table 2 identifies where consumer quality ratings were significantly different between grades and where the more highly graded meat gave better eating quality (o) or not $(\sim)$. No system was perfect. Beef passing the NZ and US systems delivered improved eating quality inconsistently and performed poorly for roasts. These systems do not consider hanging method and the US system focuses on marbling, which is generally much lower in European carcases. The UK Blueprint performed well only when low conformation grades were included (UKBP-C). Versions of the MSA system performed well for the greatest proportion of muscles and for both cooking methods. The inclusion of bulls (MSAB) improved performance for some muscles.

Table 2 Ability of beef eating quality systems to show differentiation between different levels of consumer satisfaction

\begin{tabular}{|c|c|c|c|c|c|c|c|c|c|c|c|c|c|}
\hline \multirow{2}{*}{$\begin{array}{l}\text { Cooking } \\
\text { Grilled }\end{array}$} & \multirow{2}{*}{$\begin{array}{l}\text { Muscle } \\
\text { Striploin - anterior (l. dorsi) }\end{array}$} & \multicolumn{2}{|c|}{ MSA } & \multicolumn{2}{|c|}{ MSA-B } & \multicolumn{2}{|c|}{ USDA } & \multicolumn{2}{|c|}{ UKBP } & \multicolumn{2}{|c|}{ UKBP-C } & \multicolumn{2}{|c|}{ NZQM } \\
\hline & & $* * *$ & $\mathrm{O}$ & bx & & ns & $\sim$ & $*$ & $\mathrm{O}$ & $* * *$ & $\mathrm{O}$ & ns & $\sim$ \\
\hline & Striploin - mid (l. dorsi) & $* * *$ & $\sim$ & ns & $\sim$ & $* *$ & $\mathrm{o}$ & ns & $\sim$ & ns & $\sim$ & $* * *$ & $\mathrm{O}$ \\
\hline & Striploin - posterior (l. dorsi) & $* * *$ & $\mathrm{O}$ & $* *$ & $\mathrm{O}$ & $* * *$ & $\mathrm{O}$ & ns & $\mathrm{O}$ & $* * *$ & $\mathrm{O}$ & $* * *$ & $\mathrm{O}$ \\
\hline & Rump flat (biceps femoris) & $*$ & $\mathrm{O}$ & $*$ & $\mathrm{O}$ & ns & $\sim$ & ns & $\sim$ & ns & $\sim$ & ns & $\mathrm{O}$ \\
\hline & Rump (gluteus medius) & $* * *$ & $\mathrm{o}$ & $* * *$ & $\mathrm{o}$ & $* * *$ & $\mathrm{O}$ & $\mathrm{ns}$ & $\mathrm{O}$ & $* * *$ & $\mathrm{O}$ & $*$ & $\mathrm{o}$ \\
\hline & Rump (gluteus medius eye) & ns & $\mathrm{O}$ & $*$ & $\mathrm{O}$ & $*$ & $\mathrm{O}$ & $* *$ & $\mathrm{O}$ & $* *$ & $\mathrm{O}$ & ns & $\sim$ \\
\hline Roast & Silverside eye & ns & $\sim$ & ns & $\sim$ & ns & $\sim$ & ns & $\sim$ & ns & $\sim$ & ns & $\sim$ \\
\hline & Silverside & ns & $\mathrm{o}$ & $* *$ & $\mathrm{o}$ & ns & $\sim$ & $*$ & $\mathrm{o}$ & $*$ & $\mathrm{O}$ & ns & $\mathrm{O}$ \\
\hline & Rump (gluteus medius eye) & $* * *$ & $\mathrm{O}$ & $* * *$ & $\mathrm{O}$ & $* * *$ & $\mathrm{O}$ & ns & $\mathrm{O}$ & $* * *$ & $\mathrm{O}$ & ns & $\mathrm{O}$ \\
\hline & Topside (semimembranosus) & $* * *$ & $\mathrm{O}$ & bx & & ns & $\sim$ & $*$ & $\mathrm{O}$ & $* * *$ & $\mathrm{O}$ & $*$ & $\mathrm{O}$ \\
\hline
\end{tabular}

Statistical significance of distribution: $\mathrm{ns}, *=\mathrm{P}<0.05, * *=\mathrm{P}<0.01$, $* * *=\mathrm{P}<0.001$; direction of eating quality indicated by: o where higher grades received better consumer scores or $\sim$ no consistent relationship; bx $=$ no bulls tested

Conclusions Of the quality assurance systems tested, the standard MSA system performed best for NI beef before amendment. Both MSA and MLC Blueprint performed well with amendments. MSA allows grading of individual muscles which improves versatility.

\section{References}

Farmer, L. J. et al. 2009. Proceedings 55 ${ }^{\text {th }}$ Int. Congress on Meat Science and Technology, Copenhagen: PE7.34 